\title{
Safety of coiling with stent placement for the treatment of ruptured wide-necked intracranial aneurysms: a contemporary cohort study in a high-volume center after improvement of skills and strategy
}

\author{
*Qiao Zuo, MD, Pengfei Yang, MD, Nan Lv, MD, Qinghai Huang, MD, Yu Zhou, MD, \\ Xiaoxi Zhang, MD, Guoli Duan, MD, Yina Wu, MD, Yi Xu, MD, Bo Hong, MD, Rui Zhao, MD, \\ Qiang Li, MD, Yibin Fang, MD, Kaijun Zhao, MD, Dongwei Dai, MD, and Jianmin Liu, MD \\ Department of Neurosurgery, Changhai Hospital, Second Military Medical University, Shanghai, China
}

OBJECTIVE The authors compared the contemporary perioperative procedure-related complications between coiling with stent placement and coiling without stent placement for acutely ruptured aneurysms treated in a single center after improvement of interventional skills and strategy.

METHODS In an institutional review board-approved protocol, 133 patients who underwent coiling with stent placement and 289 patients who underwent coiling without stent placement from January 2012 to December 2014 were consecutively reviewed retrospectively. Baseline characteristics, procedure-related complications and mortality rate, angiographic follow-up results, and clinical outcomes were compared between the two groups. Univariate analysis and logistic regression analysis were performed to determine the association of procedure-related complications of coiling with stent placement with potential risk factors.

RESULTS The coiling/stent group and coiling/no-stent group were statistically comparable with respect to all baseline characteristics except for aneurysm location $(p<0.001)$ and parent artery configuration $(p=0.024)$. The immediate embolization results and clinical outcomes between the two groups showed no significant differences $(p=0.807$ and $p=$ 0.611 , respectively). The angiographic follow-up results of the coiling in stent group showed a significant higher occlusion rate and lower recurrence rate compared with the coiling/no-stent group ( $82.5 \%$ vs $66.7 \%, 3.5 \%$ vs $14.5 \%, p=0.007)$. Procedure-related intraoperative rupture and thrombosis, postoperative early rebleeding and thrombosis, and external ventricular drainage-related hemorrhagic event occurred in 3.0\% (4 of 133), $2.3 \%$ ( 3 of 133), $1.5 \%$ (2 of 133), $0.7 \%$ (1 of 133 ), and $0.8 \%$ (1 of 133) of the coiling/stent group compared with $1.0 \%$ (3 of 289), $1.4 \%$ (4 of 289 ), $1.4 \%$ (4 of 289 ), and $0.7 \%$ (2 of 289) of the coiling/no-stent group, respectively $(p=0.288, p=0.810, p=1.000, p=0.315$, and $p=1.000$, respectively). One patient presented with coil protrusion in the group of coiling without stent. The procedure-related mortality was $1.5 \%$ (2 of 133) in the coiling/stent group and $0.7 \%$ in the coiling/no-stent group ( $p=0.796)$. Multivariable analysis showed no significant predictors for the total perioperative procedure-related complications, hemorrhagic complications, or ischemic complications.

CONCLUSIONS The perioperative procedure-related complications and mortality rate did not differ significantly between the coiling/stent group and the coiling/no-stent group for patients with acutely ruptured aneurysms. Considering the better angiographic follow-up results, coiling with stent placement might be a feasible, safe, and promising option for treatment in the acute phase of selected wide-necked ruptured intracranial aneurysms.

https://thejns.org/doi/abs/10.3171/2018.3.JNS172199

KEYWORDS ruptured intracranial aneurysm; coiling with stent placement; procedure-related complication; safety; vascular disorders

ABBREVIATIONS EVD = external ventricular drainage; $m R S=$ modified Rankin Scale.

SUBMITTED September 6, 2017. ACCEPTED March 12, 2018.

INCLUDE WHEN CITING Published online August 17, 2018; DOI: 10.3171/2018.3.JNS172199.

${ }^{*}$ Q.Z., P.Y., and N.L. contributed equally to this study. 
$\mathrm{S}$ TENT-ASSISTED coiling has been extensively used for the treatment of unruptured wide-necked intracranial aneurysms. Many studies have demonstrated that long-term effectiveness of stent-assisted coiling is significantly better than that of balloon-assisted coiling or coiling alone, and stent-assisted coiling achieved better complete occlusion rates and lower recurrence rates at follow-up..$^{11,18,19}$ Furthermore, several early studies also proved that coiling with stent placement was feasible for the treatment of acutely ruptured intracranial aneurysms, but the periprocedural safety remained controversial, $, 114,17$ and thus the 2012 AHA/ASA Guidelines for the Management of Aneurysmal Subarachnoid Hemorrhage suggested that coiling with stent placement should only be considered when less risky options have been excluded. ${ }^{5}$

With the improvement of neurointerventional devices and experiences on ruptured aneurysm embolization, several recent studies reported comparable complication rates between coiling with stent placement and coiling without stent placement on acutely ruptured aneurysms. . $^{3,4,721}$ Our previous study also showed that the angiographic and clinical outcomes of coiling with stent placement for 211 acutely ruptured aneurysm from 2000 to 2011 were comparable to those reported for coiling alone or balloon-assisted coiling techniques. ${ }^{20}$ However, these results were all based on case-series studies, leading to obvious selection bias, and a further case-control study was needed to evaluate the safety of coiling with stent placement on acutely ruptured aneurysms.

The complications of coiling with stent placement on acutely ruptured aneurysms are substantially attributed to antiplatelet medication management during the periprocedural period. ${ }^{15}$ Inadequate antiplatelet therapy might result in increased thrombotic events and excessive antiplatelet therapy might result in elevated hemorrhagic events theoretically. Therefore, it is more reasonable to make a comparison between one group with antiplatelet agent administration and another without antiplatelet agent when evaluating the periprocedural safety of coiling with stent placement on acutely ruptured aneurysms. However, several recent case-control studies compared the complication rates between stent-assisted coiling and balloon-assisted coiling on acutely ruptured aneurysms, ${ }^{3,4}$ and using the balloon-assisted coiling group as the control did not represent all cases of ruptured aneurysms in patients who did not receive antiplatelet medication and also limited the number of cases collected.

In the present study, although it is known that the ruptured aneurysms treated by coiling and stent placement are more complex than those treated by coiling alone, we still designed this challenging study to strictly evaluate the periprocedural safety of coiling with stent placement for acutely ruptured aneurysms in current years (2012-2014) while comparing with traditional coiling without stent placement in a high-volume center.

\section{Methods}

After receiving permission for institutional review board-approved protocol, 3 experienced endovascular surgeons retrospectively reviewed the angiographic and clinical data of 422 patients who underwent coiling with stent placement and coiling without stent placement for an acutely ruptured intracranial aneurysm between January 2012 and December 2014. Written informed consent was obtained from all patients.

\section{Patient Selection and Population}

The inclusion criterion for this study was acutely ruptured aneurysm, which was defined as an aneurysm treated no more than 28 days after the initial rupture. The exclusion criteria were as follows: 1) aneurysms other than saccular-that is, fusiform, traumatic, and blood blisterlike; 2) aneurysms treated with staged stent placement and stent placement alone; and 3) patients with multiple aneurysms, where there was doubt about which aneurysm had ruptured.

From January 2012 to December 2014, 641 patients with ruptured intracranial aneurysms were treated in our center. According to our criteria, a total of 422 consecutive patients with 422 ruptured aneurysms were included in this analysis (Fig. 1); 133 patients underwent coiling with stent placement and 289 patients underwent coiling without stent placement. All patients were treated within 3 days after admission, although some were transferred to our institution with a substantial delay.

\section{Procedure Technique and Medications}

Five endovascular neurosurgeons, all with more than 15 years of experience, treated these patients. All procedures were performed by using general anesthesia with a transfemoral approach and systemic heparinization, aiming for maintaining an activated clotting time of 2 to 3 times baseline.

A 6-Fr guiding catheter was inserted into the distal internal carotid artery or vertebral artery, as appropriate. All microcatheters, stents, and coils were delivered through this catheter. Stents and coils were deployed according to standard procedure, as recommended by the manufacturer. If acute thrombosis occurred during the procedure, glycoprotein IIb/IIIa inhibitor (Tirofiban; Grand Pharma) was used.

A loading dose of aspirin and clopidogrel $(300 \mathrm{mg}$ each) was administered orally or rectally intraoperatively after a decision was made to perform coiling with stent placement. In the postoperative period, patients who underwent coiling with stent placement were maintained on a dose of $100 \mathrm{mg}$ aspirin and $75 \mathrm{mg}$ clopidogrel daily for 6 weeks, followed by $100 \mathrm{mg}$ aspirin daily alone, which was continued indefinitely. Antiplatelet therapy was not given in any of the patients who received coiling without stenting either intraoperatively or postoperatively.

Additionally, surgical procedures including external ventricular drainage (EVD), ventriculoperitoneal shunt placement, and other procedures (decompressive craniectomy and/or hematoma evacuation) were performed according to each patient's clinical situation.

\section{Clinical and Angiographic Follow-Up}

All the patients underwent the first clinical follow-up at discharge, and the surviving patients were advised to 


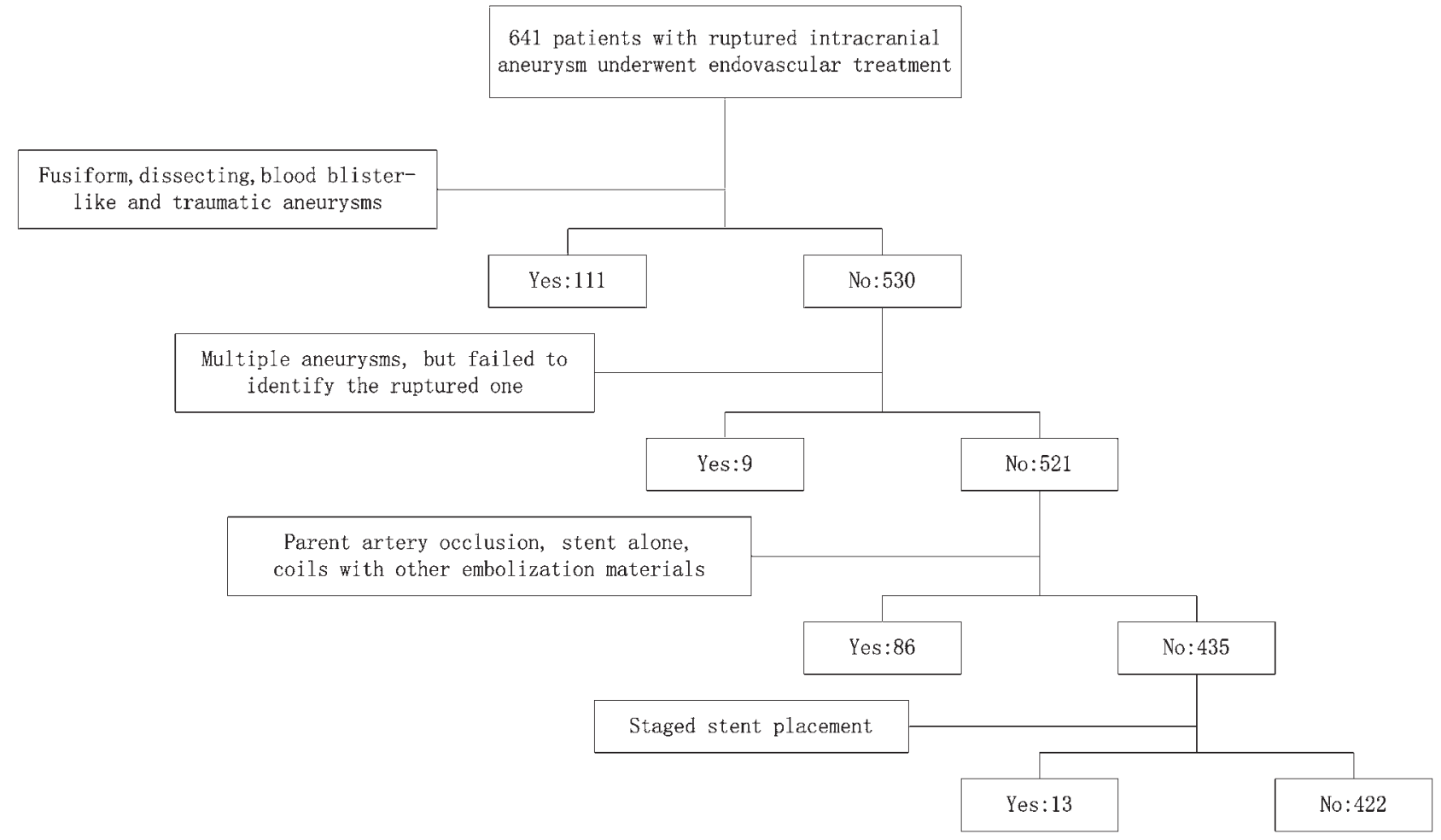

FIG. 1. Flowchart showing the process of patient selection according to the inclusion and exclusion criteria.

undergo both clinical and angiographic follow-up 3, 6, and 12 months after the treatment and annually thereafter. The clinical outcome evaluations were based on the modified Rankin Scale (mRS) scores. Good outcomes were defined as an mRS score of 0-2; poor outcomes were defined as an $\mathrm{mRS}$ score of 3-6. Procedure-related mortality and complications defined as intraprocedural thrombosis and rupture, postprocedural rupture and thrombosis, coil escape, and surgical procedure-related hemorrhagic event were recorded. The angiographic follow-up results obtained with digital subtraction angiography were classified into 4 categories when compared with the immediate embolization degree: 1) occluded, defined as no contrast material filling into the aneurysm sac; 2) improved, defined as decreased contrast material filling into the aneurysm sac; 3 ) stable, defined as unchanged contrast material filling into the aneurysm sac; or 4) recanalized, defined as increased contrast material filling into the aneurysm sac. The same two physicians who evaluated the immediate posttreatment angiographic results also evaluated the angiographic follow-up studies.

\section{Statistical Analysis}

Data are presented as mean \pm standard deviation for continuous variables and as frequency for categorical variables. Statistical analysis was carried out with unpaired ttest, Pearson chi-square test, Fisher exact test, or nonparametric test. Potential risk factors related to the procedurerelated complications (patient age and sex, Hunt and Hess score, Fisher grade, aneurysm location and size, interval from subarachnoid hemorrhage to treatment, immediate embolization degree, and surgical procedure) were categorized and analyzed (Tables 1 and 2). Univariate analysis and logistic regression were performed to determine the independent association of procedure-related complications with potential risk factors. The univariate analysis cutoff for inclusion in the logistic regression analysis was $\mathrm{p}<0.20$. The level of statistical significance was set at 0.05 . Statistical analysis was performed by using SPSS version 18.0 software.

\section{Results \\ Participants}

The coiling/stent group and coiling/no-stent group were statistically comparable with respect to age, sex, aneurysm size, interval between aneurysm rupture and treatment, Hunt and Hess grade, Fisher grade, and surgical procedure preoperatively (Table 1 ). The coiling/stent group had a higher proportion of posterior communicating artery aneurysms (51.1\% vs $23.9 \%$ ) and a lower proportion of anterior cerebral artery and anterior communicating artery aneurysms $(0.7 \%$ vs $9.0 \%, 19.3 \%$ vs $38.1 \%)$.

\section{Embolization Results and Clinical Outcomes}

Immediate complete occlusion (Raymond class I), neck residual (Raymond class II), and sac residual (Raymond class III) was achieved in 89 patients $(66.9 \%, 89$ of 133), 27 patients $(20.3 \%, 27$ of 133$)$, and 17 patients $(12.8 \%, 17$ of 133) in the coiling/stent group, respectively, and was achieved in 185 patients $(64.0 \%, 185$ of 289), 61 patients $(21.1 \%, 61 / 289)$, and 43 patients $(14.9 \%, 43$ of 289$)$ in the 
TABLE 1. Patient and aneurysm characteristics

\begin{tabular}{|c|c|c|c|}
\hline \multirow[b]{2}{*}{ Characteristic } & \multicolumn{2}{|c|}{ Coiling Group } & \multirow[b]{2}{*}{$\begin{array}{c}p \\
\text { Value }\end{array}$} \\
\hline & $\begin{array}{l}w / \text { Stent } \\
(n=133)\end{array}$ & $\begin{array}{l}\text { w/o Stent } \\
(n=289)\end{array}$ & \\
\hline Age, yrs & $58.1 \pm 11.7$ & $56.2 \pm 12.1$ & 0.130 \\
\hline \multicolumn{4}{|l|}{ Sex } \\
\hline Female & $93(69.9)$ & $182(63.0)$ & \multirow{2}{*}{0.164} \\
\hline Male & $40(30.1)$ & $107(37.0)$ & \\
\hline Aneurysm size, mm & $5.5 \pm 3.2$ & $5.0 \pm 2.5$ & 0.125 \\
\hline \multicolumn{4}{|l|}{ Location } \\
\hline ICA & $13(9.8)$ & $26(9.0)$ & \multirow{6}{*}{$<0.001$} \\
\hline PCoA & $69(51.9)$ & $69(23.9)$ & \\
\hline ACA & $1(0.8)$ & $26(9.0)$ & \\
\hline $\mathrm{ACoA}$ & $25(18.8)$ & $110(38.1)$ & \\
\hline MCA & $14(10.5)$ & $40(13.8)$ & \\
\hline PC & $11(8.3)$ & $18(6.2)$ & \\
\hline \multicolumn{4}{|c|}{ Interval btwn rupture \& treatment } \\
\hline$\leq 72 \mathrm{hrs}$ & $103(77.4)$ & $232(80.3)$ & \multirow{3}{*}{0.563} \\
\hline $3-14$ days & $25(18.8)$ & $51(17.6)$ & \\
\hline 14-28 days & $5(3.8)$ & $6(2.1)$ & \\
\hline \multicolumn{4}{|c|}{ Parent artery configuration } \\
\hline Bifurcation & $120(90.2)$ & $236(81.7)$ & \multirow{2}{*}{0.024} \\
\hline Side wall & $13(9.8)$ & $53(18.3)$ & \\
\hline \multicolumn{4}{|l|}{ Hunt \& Hess grade } \\
\hline I & $72(54.1)$ & $128(44.3)$ & \multirow{5}{*}{0.125} \\
\hline II & $29(21.8)$ & $83(28.7)$ & \\
\hline III & $19(14.3)$ & $49(17.0)$ & \\
\hline IV & $13(9.8)$ & $27(9.3)$ & \\
\hline V & $0(0.0)$ & $2(0.7)$ & \\
\hline \multicolumn{4}{|l|}{ Fisher grade } \\
\hline 0 & $0(0.0)$ & $2(0.7)$ & \multirow{5}{*}{0.876} \\
\hline 1 & $3(2.2)$ & $8(2.8)$ & \\
\hline 2 & $105(77.8)$ & $219(75.8)$ & \\
\hline 3 & $25(18.5)$ & $58(20.1)$ & \\
\hline 4 & $2(1.5)$ & $2(0.7)$ & \\
\hline Surgical procedure & $8(6.0)$ & $22(7.6)$ & 0.553 \\
\hline EVD & $7(5.3)$ & $15(5.2)$ & 0.975 \\
\hline VP shunt & $1(0.8)$ & $2(0.7)$ & 1.000 \\
\hline Others & $0(0)$ & $5(1.7)$ & 0.298 \\
\hline
\end{tabular}

$\mathrm{ACA}=$ anterior cerebral artery; $\mathrm{ACOA}=$ anterior communicating artery; $\mathrm{ICA}=$ internal carotid artery; $M C A=$ middle cerebral artery; $P C=$ posterior circulation; $\mathrm{PCOA}=$ posterior communicating artery; $\mathrm{VP}$ shunt $=$ ventriculoperitoneal shunt.

Unless indicated otherwise, data are presented as the number of patients (\%).

coiling/no-stent group, respectively, which showed no significant difference between the two groups $(\mathrm{p}=0.807)$.

Of the 408 patients who survived the initial subarachnoid hemorrhage, 348 patients with 348 aneurysms (85.3\%) underwent angiographic follow-up at least once, ranging from 3 days to 1787 days (median 391 days). Follow-up angiograms showed that 94 aneurysms $(82.5 \%, 94$ of 114) were occluded, 9 aneurysms $(7.9 \%, 9$ of 114) were
TABLE 2. Angiographic and clinical outcomes of coiling with stent placement and coiling without stent placement

\begin{tabular}{|c|c|c|c|}
\hline \multirow[b]{2}{*}{ Outcome } & \multicolumn{2}{|c|}{ Coiling Group } & \multirow{2}{*}{$\begin{array}{c}p \\
\text { Value }\end{array}$} \\
\hline & w/ Stent & w/o Stent & \\
\hline \multicolumn{4}{|c|}{ Immediate embolization result } \\
\hline Raymond class I & $89(66.9)$ & $185(64.0)$ & \multirow{3}{*}{0.807} \\
\hline Raymond class II & $27(20.3)$ & $61(21.1)$ & \\
\hline Raymond class III & $17(12.8)$ & $43(14.9)$ & \\
\hline \multicolumn{4}{|l|}{ Angiographic follow-up } \\
\hline Occluded & $94(82.5)$ & $156(66.7)$ & \multirow{4}{*}{0.007} \\
\hline Improved & $9(7.9)$ & $23(9.8)$ & \\
\hline Stable & $7(6.1)$ & $21(9.0)$ & \\
\hline Recanalized & $4(3.5)$ & $34(14.5)$ & \\
\hline \multicolumn{4}{|l|}{ Clinical follow-up } \\
\hline mRS score 0-2 & $116(87.2)$ & $257(88.9)$ & \multirow{2}{*}{0.611} \\
\hline mRS score 3-6 & $17(12.8)$ & $32(11.1)$ & \\
\hline
\end{tabular}

Unless indicated otherwise, data are presented as the number of patients (\%).

improved, 7 aneurysms $(6.1 \%, 7$ of 114) were stable, and 4 aneurysms $(3.5 \%, 4$ of 114) were recanalized in the coiling/ stent group. All parent arteries were patent without clinically significant in-stent stenosis. In the coiling/no-stent group, 156 aneurysms $(66.7 \%, 156$ of 234) were occluded, 23 aneurysms $(9.8 \%, 23$ of 234) were improved, 21 aneurysms $(9.0 \%, 21$ of 234$)$ were stable, and 34 aneurysms $(14.5 \%, 34$ of 234$)$ were recanalized. The angiographic follow-up results were significantly different between the two groups, with a higher occlusion rate and lower recurrence rate in the coiling/stent group $(p=0.007)$.

All surviving patients underwent clinical follow-up after discharge at intervals between 3 months and 59 months (median, 36 months). These evaluations showed that 116 patients $(87.2 \%, 116$ of 133) and 257 patients $(88.9 \%, 257$ of 289) had a good outcome (mRS score of 0-2) in the coiling/stent group and in the coiling/no-stent group respectively $(\mathrm{p}=0.611)$.

\section{Perioperative Procedure-Related Complications and Mortality}

Overall, perioperative procedure-related complications occurred in 11 patients $(8.3 \%, 11$ of 133) in the coiling/ stent group and in 13 patients $(4.5 \%, 13$ of 289$)$ in the coiling/no-stent group, which were comparable $(\mathrm{p}=0.120)$. Specifically, the hemorrhagic and ischemic complications of the coiling/stent group were both slightly higher than those of the coiling/no-stent group, but the differences were without statistical significance (Table 3).

For hemorrhagic complications, intraprocedural rupture, aneurysm rebleeding, and surgical procedure-related hemorrhagic events occurred in 4 patients $(3.0 \%, 4$ of 133$)$, 2 patients $(1.5 \%, 2$ of 133$)$, and 1 patient $(0.8 \%, 1$ of 133$)$ of the coiling/stent group and in 3 patients $(1.0 \%, 3$ of 289$)$, 4 patients $(1.4 \%, 4$ of 289$)$, and $2(0.7 \%, 2$ of 289$)$ patients of the coiling/no-stent group, respectively $(\mathrm{p}=0.288, \mathrm{p}=$ 1.000 , and $p=1.000$, respectively; Table 3 ). All 3 patients who suffered a surgical procedure-related hemorrhagic 
TABLE 3. Perioperative procedure-related complications

\begin{tabular}{cccc}
\hline & \multicolumn{2}{c}{ Coiling Group } & \\
\cline { 2 - 3 } Complication & $\begin{array}{c}\text { w/ Stent } \\
(\mathrm{n}=133)\end{array}$ & $\begin{array}{c}\text { w/o Stent } \\
(\mathrm{n}=289)\end{array}$ & $\begin{array}{c}\mathrm{p} \\
\text { Value }\end{array}$ \\
\hline Procedure-related complications & $11(8.3)$ & $13(4.5)$ & 0.120 \\
\hline Hemorrhagic & $7(5.3)$ & $8(2.8)$ & 0.316 \\
\hline Intraprocedural rupture & $4(3.0)$ & $3(1.0)$ & 0.288 \\
\hline Aneurysm rebleeding & $2(1.5)$ & $4(1.4)$ & 1.000 \\
\hline $\begin{array}{c}\text { Procedure-related hemor- } \\
\text { rhagic event }\end{array}$ & $1(0.8)$ & $2(0.7)$ & 1.000 \\
\hline Ischemic & $4(3.0)$ & $4(1.4)$ & 0.452 \\
\hline Intraprocedural thrombosis & $3(2.3)$ & $4(1.4)$ & 0.810 \\
\hline Postprocedural thrombosis & $1(0.7)$ & $0(0.0)$ & 0.315 \\
\hline Coil protrusion & $0(0.0)$ & $1(0.3)$ & 1.000 \\
\hline Procedure-related mortality & $2(1.5)$ & $2(0.7)$ & 0.796 \\
\hline
\end{tabular}

Unless indicated otherwise, data are presented as the number of patients (\%).

event underwent an EVD procedure. In the coiling/nostent group, 1 patient suffered both aneurysm rebleeding and an EVD-related hemorrhagic event.

For ischemic complications, intraprocedural thrombosis and postprocedural thrombosis occurred in 3 patients (2.3\%, 3 of 133) and 1 patient $(0.7 \%, 1$ of 133$)$ of the coiling/stent group and in 4 patients $(1.4 \%, 4$ of 289$)$ and 0 patient of the coiling/no-stent group, respectively $(\mathrm{p}=$ 0.810 and $p=0.315$, respectively; Table 3). All 8 patients with ischemic events intraoperatively or postoperatively received intravenous thrombolysis with the glycoprotein IIb/IIIa inhibitor Tirofiban in both the coiling/stent group and coiling without stent group. Most of these patients $(87.5 \%, 7$ of 8$)$ achieved a good clinical outcome on discharge (mRS 0-2). One patient in the coiling/no-stent group $(0.3 \%, 1$ of 289$)$ suffered coil protrusion into the parent artery without clinical symptoms.

Among the patients who suffered procedure-related complications, 2 of them died in the coiling/stent group, and 2 of them died in the coiling/no-stent group. In the coiling/stent group, one patient suffered from an intraprocedural rupture and the other patient suffered from aneurysm rebleeding. In the coiling/no-stent group, the 2 patients died due to aneurysm rebleeding. The procedurerelated mortality rate between the coiling/stent group and coiling/no-stent group was also comparable (1.5\% vs $0.7 \%, \mathrm{p}=0.796$; Table 3).

\section{Multivariate Analysis for Risk Factors of Procedure-Related Complications}

The following factors were tested as predictors of complications: patient age, sex, Hunt and Hess grade, Fisher grade, history of hypertension, history of diabetes, history of coronary heart disease, smoking history, other cerebrovascular disease, aneurysm location, aneurysm size, treatment interval, treatment strategy, immediate embolization degree, and surgical procedure. Univariate analysis showed that the aneurysm location $(\mathrm{p}=0.144)$ and immediate embolization degree $(\mathrm{p}=0.031)$ were associated with total procedure-related complications; the aneurysm location $(\mathrm{p}=0.073)$, immediate embolization degree $(\mathrm{p}=$ $0.080)$, and treatment interval $(\mathrm{p}=0.180)$ were associated with hemorrhagic procedure-related complications; and the aneurysm location $(\mathrm{p}=0.101)$, patients with hypertension $(\mathrm{p}=0.09)$, patients with diabetes $(\mathrm{p}=0.17)$, and $\mathrm{pa}-$ tients with smoking history $(\mathrm{p}=0.17)$ were associated with ischemic procedure-related complications. However, multivariable analysis showed no predictors for the hemorrhagic, ischemic, or total procedure-related complications.

\section{Discussion}

In this single-center retrospective cohort study, both procedure-related hemorrhagic and ischemic complication rates were slightly higher in the coiling/stent group than they were in the coiling/no-stent group, but they are still comparable. The multivariate analysis showed coiling with stent placement was not a predictor for hemorrhagic, ischemic, or total complications of acutely ruptured aneurysms. These results suggest that coiling with stent placement is a reasonable choice with comparable safety of coiling without stent placement for the treatment of selected acutely ruptured intracranial aneurysms.

The results of this study are in line with those reported by Chitale et al., with 44 cases of stent-assisted coiling and 40 cases of balloon-assisted coiling for the treatment of ruptured aneurysms, which showed that the procedural complications were similar between the two groups. ${ }^{4} \mathrm{~A}$ recent study with 65 cases of stent-assisted coiling and 32 cases of balloon-assisted coiling also showed that periprocedural complications did not differ between the two different treatment strategies for ruptured wide-necked aneurysms. ${ }^{3}$ However, a recent multicenter retrospective cohort study with 23 cases of coiling with stent and 108 cases of coiling alone reported the aneurysm rebleeding rate in the coiling/stent group was significantly higher than that of the coiling-alone group for ruptured aneurysms treatment $(17.4 \%$ vs $1.9 \%, \mathrm{p}<0.007)$ probably because of incomplete aneurysm occlusion, without statistical difference of intraprocedural rupture or procedure-related ischemic complication rate. ${ }^{21}$

In a previous study, we reported a single-center, 11-year experience (from 2000 to 2011) of the safety of coiling with stent placement on 211 acutely ruptured wide-necked intracranial aneurysms. ${ }^{20}$ Compared to the previous results, both the hemorrhagic and ischemic events showed a declined trend (hemorrhagic complication rate $5.3 \%$ vs $6.2 \%$; ischemic complication rate $3.0 \%$ vs $7.6 \%$, respectively), and no predictors were found for procedure-related complications in the present study. This may be owing to the reasons as follow: 1) There is a changing strategy of periprocedural antiplatelet medication. We stopped using low-molecular-weight heparin and changed the dualantiplatelet strategy from a high dosage (aspirin $300 \mathrm{mg}$ and clopidogrel $75 \mathrm{mg}$ daily) to a low dosage (aspirin 100 $\mathrm{mg}$ and clopidogrel $75 \mathrm{mg}$ daily) after the procedure since 2010, and Tirofiban was used in a small dose cautiously (semidose of the standard strategy in acute myocardial infarction) when an ischemic event occurred during the procedure. A recent systematic review showed that com- 
plications of stent-assisted coiling for ruptured aneurysms may be affected by the method of antiplatelet administration..$^{15}$ Dual-antiplatelet medication with aspirin and clopidogrel $^{12,16}$ and monoantiplatelet therapy ${ }^{1,2,6}$ have all been proved to be feasible for perioperative use on acutely ruptured aneurysms, and there is little consensus regarding the appropriate timing and dosage for antiplatelet agent administration, so further studies are required to establish the guidelines for perioperative antiplatelet strategy on stentassisted coiling for acutely ruptured aneurysms. 2) There is a changing strategy of case selection of coiling with stent placement in ruptured aneurysms. Coiling with stent placement for patients with ruptured aneurysms on middle cerebral artery bifurcation or anterior communicating artery was avoided as much as possible after the previous study showed that aneurysms in these two locations were more likely to have a higher incidence of complications. ${ }^{20}$ Several studies also showed that stent-assisted coiling of anterior communicating artery aneurysms $\mathrm{s}^{7,13}$ and middle cerebral artery aneurysms ${ }^{10}$ had a higher complication rate than coiling alone. Therefore coiling with stent placement should be more strictly considered and used in the ruptured aneurysms on these two locations. 3) Immediate occlusion rates after procedures have improved. With the improvement of skills and experience with coiling and stent placement in our center, and the improvement of interventional embolization devices and stent deployment skills, such as smaller and softer coils, modified hydrocoils, and semi-deployment technique, the immediate aneurysm occlusion rate has increased compared to the previous rate ( $66.9 \%$ vs $45.5 \%$, respectively), which is considered an important predictor of early repeat bleeding., ${ }^{8,21}$ For these reasons, the procedure-related complication rate of coiling and stent placement for acutely ruptured aneurysms from 2012 to 2014 has decreased compared with the cases from 2000 to 2011 in our center.

The major limitation of this study is that the aneurysm characteristics between the coiling/stent group and the coiling/no-stent group were not comparable, especially the differences in aneurysm neck and parent artery configuration of the two cohorts. This is due to the design of the study, which aimed to strictly evaluate the safety of coiling with stent placement in acutely ruptured aneurysms by comparing the groups with and without antiplatelet administration. Additionally, the statistical power was 0.287 for detecting the difference of the procedure-related complication rate between the two groups, which is another major limitation of this study. The low power is attributed to the low complication rate and the limited sample size. Given the retrospective design of this study, a prospective study with a larger sample size should be done to compare the small differences in the procedure-related complications between the two groups.

\section{Conclusions}

The perioperative procedure-related complication rate for coiling and stent placement did not differ significantly from that of coiling without stent placement in acutely ruptured intracranial aneurysms, and it also showed a decreasing trend when compared with the data from 2000 to
2011 in our center. These results suggest that coiling with stent placement may be a feasible, safe, and promising option for the treatment of selected wide-necked acutely ruptured aneurysms.

\section{Acknowledgments}

This study was supported by the National Research and Development Project of Key Chronic Diseases of China (GN2016YFC1300700), the National Health and Family Planning Commission Stroke Prevention and Treatment Foundation, China (GN-2016R0012), and the National Natural Science Foundation of China (81471189, 81701136).

\section{References}

1. Amenta PS, Dalyai RT, Kung D, Toporowski A, Chandela S, Hasan D, et al: Stent-assisted coiling of wide-necked aneurysms in the setting of acute subarachnoid hemorrhage: experience in 65 patients. Neurosurgery 70:1415-1429, 2012

2. Bechan RS, Sprengers ME, Majoie CB, Peluso JP, Sluzewski M, van Rooij WJ: Stent-assisted coil embolization of intracranial aneurysms: complications in acutely ruptured versus unruptured aneurysms. AJNR Am J Neuroradiol 37:502507, 2016

3. Cai K, Zhang Y, Shen L, Ni Y, Ji Q: Comparison of stentassisted coiling and balloon-assisted coiling in the treatment of ruptured wide-necked intracranial aneurysms in the acute period. World Neurosurg 96:316-321, 2016

4. Chitale R, Chalouhi N, Theofanis T, Starke RM, Amenta $\mathrm{P}$, Jabbour P, et al: Treatment of ruptured intracranial aneurysms: comparison of stenting and balloon remodeling. Neurosurgery 72:953-959, 2013

5. Connolly ES Jr, Rabinstein AA, Carhuapoma JR, Derdeyn CP, Dion J, Higashida RT, et al: Guidelines for the management of aneurysmal subarachnoid hemorrhage: a guideline for healthcare professionals from the American Heart Association/American Stroke Association. Stroke 43:1711-1737, 2012

6. Edwards NJ, Jones WH, Sanzgiri A, Corona J, Dannenbaum M, Chen PR: Antiplatelet therapy for the prevention of pericoiling thromboembolism in high-risk patients with ruptured intracranial aneurysms. J Neurosurg 127:1326-1332, 2017

7. Fan L, Tan X, Xiong Y, Zheng K, Li Z, Liu D, et al: Stent-assisted coiling versus coiling alone of ruptured anterior communicating artery aneurysms: A single-center experience.

Clin Neurol Neurosurg 144:96-100, 2016

8. Fleming JB, Hoh BL, Simon SD, Welch BG, Mericle RA, Fargen KM, et al: Rebleeding risk after treatment of ruptured intracranial aneurysms. J Neurosurg 114:1778-1784, 2011

9. Golshani K, Ferrel A, Lessne M, Shah P, Chowdhary A, Choulakian A, et al: Stent-assisted coil emboilization of ruptured intracranial aneurysms: A retrospective multicenter review. Surg Neurol Int 3:84, 2012

10. Gory B, Rouchaud A, Saleme S, Dalmay F, Riva R, Caire $\mathrm{F}$, et al: Endovascular treatment of middle cerebral artery aneurysms for 120 nonselected patients: a prospective cohort study. AJNR Am J Neuroradiol 35:715-720, 2014

11. Hong Y, Wang YJ, Deng Z, Wu Q, Zhang JM: Stent-assisted coiling versus coiling in treatment of intracranial aneurysm: a systematic review and meta-analysis. PLoS One 9:e82311, 2014

12. Lodi YM, Latorre JG, El-Zammar Z, Swarnkar A, Deshaies E, Fessler RD: Stent assisted coiling of the ruptured wide necked intracranial aneurysm. J Neurointerv Surg 4:281286, 2012

13. O'Neill AH, Chandra RV, Lai LT: Safety and effectiveness of microsurgical clipping, endovascular coiling, and stent assisted coiling for unruptured anterior communicating artery 
aneurysms: a systematic analysis of observational studies. J Neurointerv Surg 9:761-765, 2017

14. Piotin M, Blanc R, Spelle L, Mounayer C, Piantino R, Schmidt PJ, et al: Stent-assisted coiling of intracranial aneurysms: clinical and angiographic results in 216 consecutive aneurysms. Stroke 41:110-115, 2010

15. Ryu CW, Park S, Shin HS, Koh JS: Complications in stentassisted endovascular therapy of ruptured intracranial aneurysms and relevance to antiplatelet administration: a systematic review. AJNR Am J Neuroradiol 36:1682-1688, 2015

16. Shimamura N, Naraoka M, Matsuda N, Ohkuma H: Safety of preprocedural antiplatelet medication in coil embolization of ruptured cerebral aneurysms at the acute stage. Interv Neuroradiol 20:413-417, 2014

17. Tähtinen OI, Vanninen RL, Manninen HI, Rautio R, Haapanen A, Niskakangas T, et al: Wide-necked intracranial aneurysms: treatment with stent-assisted coil embolization during acute ( $<72$ hours) subarachnoid hemorrhage-experience in 61 consecutive patients. Radiology 253:199-208, 2009

18. Wang F, Chen X, Wang Y, Bai P, Wang HZ, Sun T, et al: Stent-assisted coiling and balloon-assisted coiling in the management of intracranial aneurysms: A systematic review \& meta-analysis. J Neurol Sci 364:160-166, 2016

19. Yang H, Sun Y, Jiang Y, Lv X, Zhao Y, Li Y, et al: Comparison of stent-assisted coiling vs coiling alone in 563 intracranial aneurysms: safety and efficacy at a high-volume center. Neurosurgery 77:241-247, 2015

20. Yang P, Zhao K, Zhou Y, Zhao R, Zhang L, Zhao W, et al: Stent-assisted coil placement for the treatment of 211 acutely ruptured wide-necked intracranial aneurysms: a single-center 11-year experience. Radiology 276:545-552, 2015

21. Zhao B, Tan X, Yang H, Zheng K, Li Z, Xiong Y, et al: Stentassisted coiling versus coiling alone of poor-grade ruptured intracranial aneurysms: a multicenter study. J Neurointerv Surg 9:165-168, 2017

\section{Disclosures}

The authors report no conflict of interest concerning the materials or methods used in this study or the findings specified in this paper.

\section{Author Contributions}

Conception and design: Liu, Zuo, Yang, Lv, Huang, Xu, Hong. Acquisition of data: Liu, Zuo, Yang, Zhou, Zhang, Duan, Wu. Analysis and interpretation of data: Liu, Zuo, Yang, Lv, Huang, $\mathrm{Xu}$, Hong, R Zhao, Li, Fang, K Zhao, Dai. Drafting the article: Zuo, Yang. Critically revising the article: Liu, Zuo, Yang, Lv, Huang, $\mathrm{Xu}$, Hong. Reviewed submitted version of manuscript: Liu, Zuo, Yang, Lv. Approved the final version of the manuscript on behalf of all authors: Liu. Statistical analysis: Zuo, Zhou, Zhang. Administrative/technical/material support: Liu, Yang, Huang, Xu, Hong. Study supervision: Liu, Zuo, Yang, Huang.

\section{Correspondence}

Jianmin Liu: Changhai Hospital, Second Military Medical University, Shanghai, China.liu118@vip.163.com. 